\title{
Flow-induced crystallization studied in the RheoDSC device: quantifying the importance of edge effects.
}

\author{
Peter C. Roozemond, Martin van Drongelen, Leander Verbelen, \\ Peter van Puyvelde, and Gerrit W.M. Peters
}

\begin{abstract}
Flow-induced crystallization is investigated through short-term shear flow experiments on poly(1-butene) in the RheoDSC device. We demonstrate that the DSC signal shows contributions from spherulitic morphology in the center of the sample and oriented structures at the edge of the sample, the latter seemingly being induced by edge effects at the free surface. We show that, although small in terms of volume, the crystallization at the edge has a dominating influence on the measured rheology. We show how these kinds of effects can be recognized in stand-alone rheometric studies of flow-induced crystallization.
\end{abstract}

\section{Introduction}

Rheometry is a widely used tool to study Flow-Induced Crystallization (FIC), because this technique possesses the combined possibilities to apply flow at elevated temperatures and monitor structure development (via the rheology) $[1,2]$. Such studies have been performed on for example isotactic polypropylene (iPP) [2--5], polyethylene (PE) [1, 6], isotactic poly(1-butene) (iP1B) [7--10], and poly(lactic acid) (PLA) [11], either using the short-term shear protocol [12], cf. refs. [2,3], or using continuous shear $[7,10]$. It has even been shown that using an appropriate model to link rheological properties and crystalline structure [13, 14], one can extract nucleation density after short-term shear from such experiments [3, 4], thus providing an alternative for studies with in-situ optical microscopy.

The recently developed RheoDSC [15--17], a device that allows in-situ Differential Scanning Calorimetry (DSC) on rheological measurements, has proven to be a valuable augmentation to these types of experiments. The DSC signal gives a direct measure for the progress of crystallization via latent heat release. The device was used for the study of shear induced crystallization during continuous shear flow at mild rates. Moreover, it was shown that even a weak oscillatory shear can significantly enhance crystallization kinetics [18]. Furthermore, it was used for the validation of a model that calculates rheological properties from crystalline volume fraction [9].

In this work we demonstrate the applicability of the RheoDSC device to short-term shear flow experiments. Simultaneously performing DSC and rheometry, we identified crystallization of the bulk of the sample in spherulitic morphology and oriented crystallization in a small part of the sample, presumably induced by edge effects near the free surface. It is shown that, although by volume the fraction of material crystallized in this morphology is rather small, the influence on the measured rheology is tremendous. Our results have important implications for the analysis of stand-alone rheometric studies of FIC: if edge effects occur (i.e. at high shear rates), the rheometric signal might be representative for material near the edge of the sample only, instead of the whole volume. We show how these effects can be recognized in the rheometric data. 


\section{Experimental}

An isotactic poly(1-butene), grade $\mathrm{PB} 400\left(\mathrm{M}_{w}=176 \mathrm{~kg} / \mathrm{mol}, \mathrm{M}_{w} / \mathrm{M}_{n}=5.7\right.$, isotacticity=98.8\%) [19] was subjected to flow and subsequently analyzed during isothermal crystallization in the prototype RheoDSC device. This instrument was constructed starting from two stand-alone commercial instruments: a Q200o Tzero ${ }^{\mathrm{TM}} \mathrm{DSC}$ (TA Instruments) and an AR-G2 rheometer (TA Instruments) fitted with plate-plate geometry. The DSC apparatus uses the Tzero ${ }^{\top M}$ technology, which is important for the correction of the thermal resistances and capacitances of the various heat flow paths in the cell caused by the modification of the calorimetric cell environment. The AR-G2 rheometer is a sensitive instrument opening the range of ultra-low torques, which is of importance as the diameter of the measurement geometry is chosen to be of comparable dimension as the DSC sensor ( $5 \mathrm{~mm}$ radius, $350 \mu \mathrm{m}$ sample height, $6.5 \mathrm{mg}$ sample mass). For more details the reader is referred to ref. [15]. Both the rheometric and thermal signals of the RheoDSC device have been validated, as reported by refs. [15--17].

Before each experiment, thermo-mechanical history was erased by keeping the sample at $200{ }^{\circ} \mathrm{C}$ for 10 minutes. The sample was cooled down from $200{ }^{\circ} \mathrm{C}$ to $92{ }^{\circ} \mathrm{C}$ at $10{ }^{\circ} \mathrm{C} / \mathrm{min}$, after which it was kept at $92{ }^{\circ} \mathrm{C}$ for 5 minutes in order to reach thermal equilibrium of the setup. After this equilibration time, the sample was subjected to a shear pulse with a duration of $10 \mathrm{~s}$ with shear rates from $5 \mathrm{~s}^{-1}$ to $30 \mathrm{~s}^{-1}$ with increments of $5 \mathrm{~s}^{-1}$. The heat flow and rheology were monitored during subsequent isothermal crystallization until crystallization was complete.

\section{Results}

\subsection{DSC}

The heat flow after baseline subtraction [18] is shown in Fig. 1(a). Time $t=0$ is the end of flow. For low shear rates we observe an exothermal crystallization peak with a maximum around 300 s. For higher shear rates the maximum shifts to shorter times. As this peak is already present at low shear rate, where no orientation is expected $[7,19]$, we assign this peak to crystallization of spherulites. The typical time scale of crystallization of such a morphology can be estimated using the Avrami equation for isothermal crystallization of heterogeneously nucleated spherulites[20],

$$
\xi=1-\exp \left(-\frac{4 \pi}{3} N_{\mathrm{sph}} G^{3} t^{3}\right) .
$$

With $\xi$ space filling, $N_{\mathrm{sph}}$ the number of spherulites and $G$ the crystal growth rate. At $92{ }^{\circ} \mathrm{C}$, the growth rate of this material $G=0.1 \mu \mathrm{m} / \mathrm{s}$ [21] and a typical nucleation density for these shear rates $N_{\mathrm{sph}} \approx 10^{13} \mathrm{~m}^{-3}$ [22]. Thus we indeed find a crystallization half time of $\sim 250 \mathrm{~s}$, in accordance with what we see in the experiments. This confirms that this peak originates from crystallization of spherulites.

For higher shear rates, a second peak appears at short times (maximum at $t \approx 1 \mathrm{~s}$ ), and heat flow shortly after flow is strongly increased with respect to the value in quiescent conditions. We ascribe the latter to shear heating, which increases the temperature of the sample during flow. This additional heat is slowly dissipated after flow, which shows in the DSC signal. To account for this effect and isolate the contributions from crystallization, we subtracted an additional baseline from the DSC signal to account for the viscous dissipation. This baseline, following ref. [18], is of the form

$$
\Phi(t)=\Phi_{0} \exp (-t / \lambda),
$$

with $\Phi(t)$ the heat flow due to stored energy from shear heating, and $\Phi_{0}$ and $\lambda$ adjustable parameters that were determined per experiment. The typical time scale is between 13.7 and 19.2 


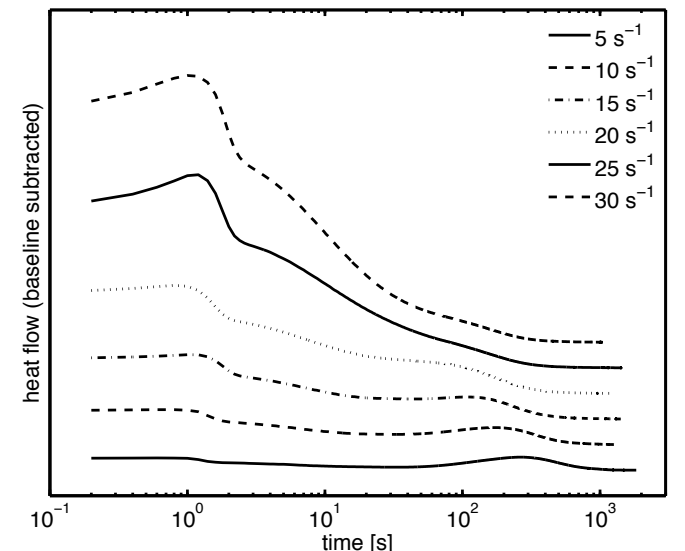

(a)

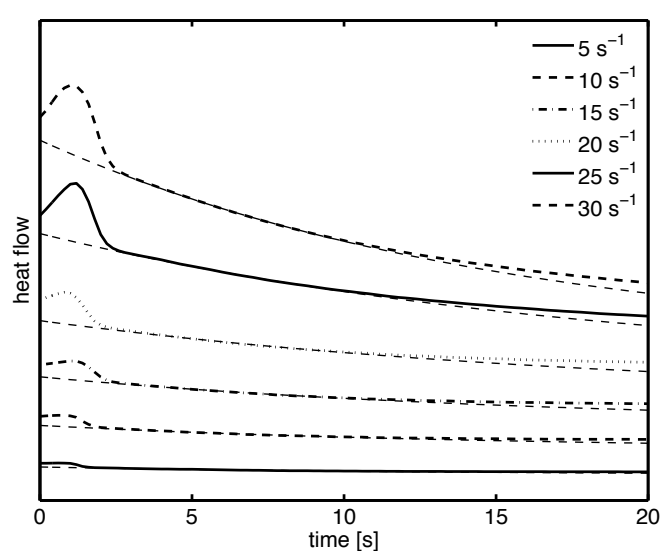

(b)

Figure 1: Heat flow for all experiments after baseline subtraction. The thin dashed lines in (b) show the baseline representing dissipation of stored energy from shear heating (Eq. 2). Signals are shifted in vertical direction for clarity.

seconds for all experiments, $\Phi_{0}$ increased with shear rate. This additional baseline is shown for all experiments in Fig. 1(b). In Appendix A we show that the trend observed in the parameters for this baseline correspond well to the expected trends for shear heating. 
Figure 2 shows the DSC signal after subtraction of the contribution due to viscous dissipation. Now we more clearly observe, with increasing shear rate, the appearance of the second peak at short times. We consider this peak to be the signature of crystallization of shish-kebabs. An estimate with the Avrami equation for crystallization of shish-kebabs,

$$
\xi=1-\exp \left(-2 \pi L G^{2} t^{2}\right),
$$

with $L$ the density of shish (typically $10^{12} \mathrm{~m} / \mathrm{m}^{3}$ for isotactic polypropylene, which, at least in the point-like regime, shows comparable numbers to $\mathrm{P} 1 \mathrm{~B}[23,24]$ ), indeed gives a half crystallization time of $\sim 3 \mathrm{~s}$. Crystal growth rate, which does not depend on morphology, is again denoted by $G$.

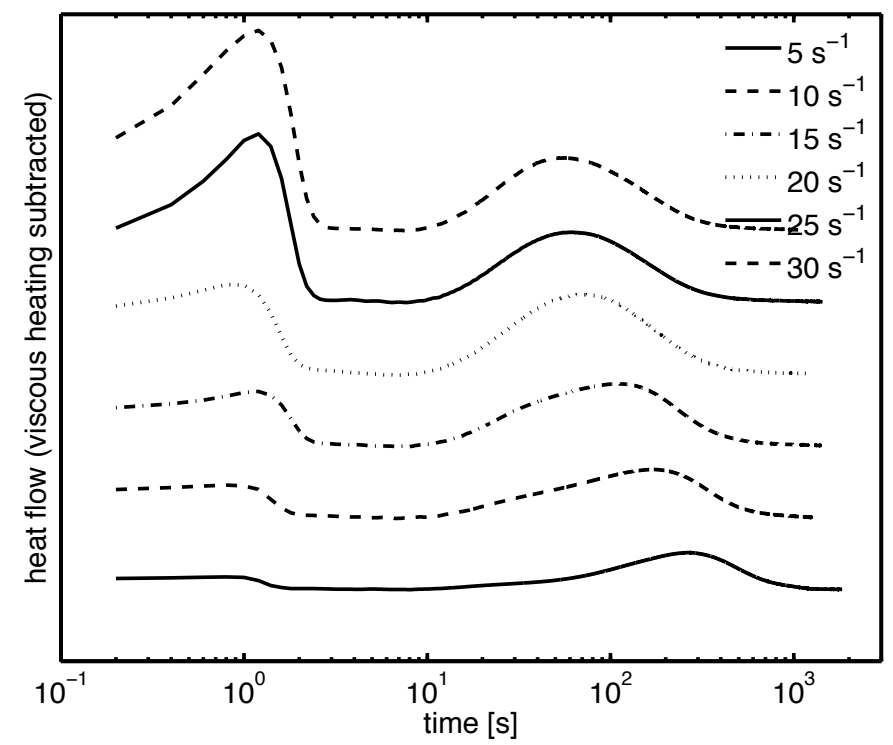

Figure 2: Heat flow for all experiments after subtraction of the heat flow due to stored energy from shear heating. Signals are shifted in vertical direction for clarity.

Because the two peaks (from spherulitic and shish-kebabs morphology) are well separated in the time domain (between 3 and 10 seconds the heat flow is zero in all experiments), we can easily determine how much of the sample crystallizes in which morphology. To do so, we calculated the total heat released by each of the processes by time integration over the appropriate time intervals.

$$
\begin{aligned}
\Delta H_{\text {shish-kebab }} & =\int_{0}^{t_{1}} w(t) \mathrm{d} t \\
\Delta H_{\text {spherulites }} & =\int_{t_{1}}^{t_{\infty}} w(t) \mathrm{d} t .
\end{aligned}
$$

With $t_{\infty}=2000 \mathrm{~s}$ and $t_{1}$ is the time where shish-kebabs have fully crystallized and the space filling of spherulites is still negligible, $t_{1}=5 \mathrm{~s}$. The heat flow measured by DSC after subtraction of baseline and contribution from shear heating is denoted by $w(t)$. The results are shown in Fig. 3 (a). The total heat release decreases with increasing shear rate, from $25 \mathrm{~J} / \mathrm{g}$ at the lowest shear rate to $18 \mathrm{~J} / \mathrm{g}$ at the highest shear rate. This result is hard to explain. Possibly the fast growth of crystallites leads to more defects, which are perfected during a secondary crystallization process 


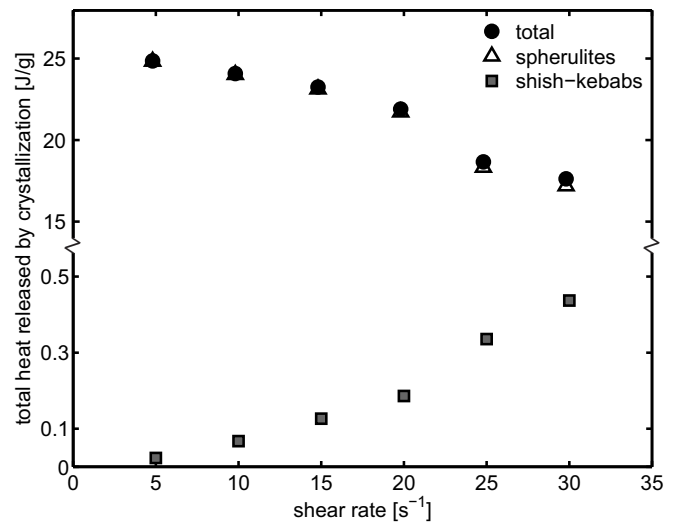

(a)

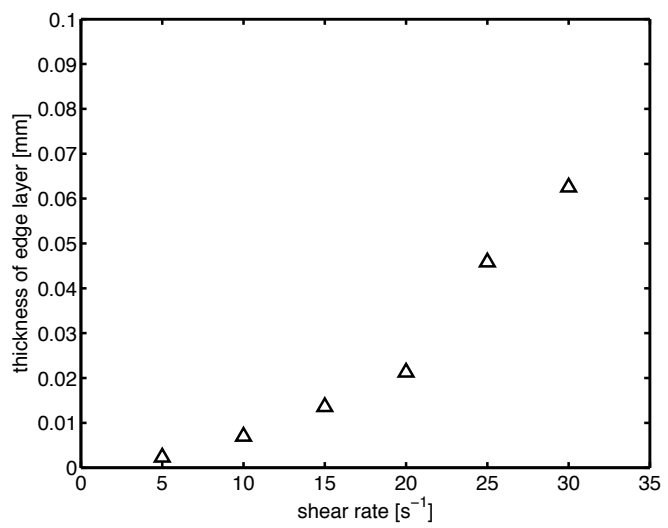

(b)

Figure 3: (a) Total heat released by crystallization. (b) Thickness of domain with shish-kebabs.

which is undetectable by DSC, or there are some inaccuracies in the determination of the baselines.

Figure 3(a) further shows that, although increasing with shear rate, the amount of shish-kebab remains very low compared to the amount of spherulites for all flow conditions. If we assume that the heat of fusion per unit volume is equal for spherulites and shish-kebabs, at the highest shear rate still only $3 \%$ of the total volume is filled with shish-kebabs. Therefore the sample is not separated into regions of isotropic and oriented crystallization by a boundary at a critical shear rate (which depends on radius in parallel plate geometry), like in the work of Mykhaylyk and co-workers $[25,26]$. In that case, having a small amount of shish-kebabs at $10 \mathrm{~s}^{-1}$ would mean that, at the highest shear rate, $1-(10 / 30)^{2}=89 \%$ of the sample would be highly oriented. Instead, oriented crystallization takes place in a layer near the edge of the sample, the thickness of which only increases slightly with shear rate. We can calculate the thickness of the layer with shish-kebabs from

$$
d=R\left(1-\sqrt{\frac{\Delta H_{\text {spherulites }}}{\Delta H_{\text {total }}}}\right)
$$

with $R=5 \mathrm{~mm}$ the radius of the sample, and $\Delta H$ from equation 4. Figure 3(b) shows the thickness of this layer, which turns out to be quite thin, $60 \mu \mathrm{m}$ at the highest shear rate.

We believe that the oriented morphology in this layer is a result of edge effects. The edges of a sample in the RheoDSC device can not be cleaned like in a conventional rheometer, which possibly induces some disturbances in the flow field near the free surface. Although the effect on the overall crystallization kinetics is quite small, this effect can strongly influence the measured rheology. We will come back to this in section 3.3.

\subsection{Crystallization kinetics}

Figure 4(a) shows the evolution of space filling of spherulites averaged over the volume of the sample with isotropic morphology, given by

$$
\xi(t)=\frac{1}{\Delta H_{\text {spherulites }}} \int_{t_{1}}^{t} w\left(t^{\prime}\right) d t^{\prime},
$$

where $\Delta H_{\text {spherulites }}$ was calculated from Eq. 4 and again $t_{1}=5 \mathrm{~s}$. As expected, the speed at which crystallization progresses increases with increasing shear rate, because flow strongly increases 
the nucleation density. Unfortunately we can not extract quantitative information about the nucleation density from these data, because, being governed by the shear rate, nucleation density depends on radial position. Therefore, the measured space filling gives an average over the whole sample. This also becomes apparent if we make an Avrami plot (Fig. 4(b)) of these data, which should give a slope of 3 for crystallization of spherulites. Because the nucleation density is not homogeneous throughout the sample, the usual Avrami analysis (in which nucleation density is constant) does not apply, and we observe slopes between 1 and 2. In Appendix B it is demonstrated that a nonhomogeneous nucleation density can indeed result in Avrami exponents in this range.

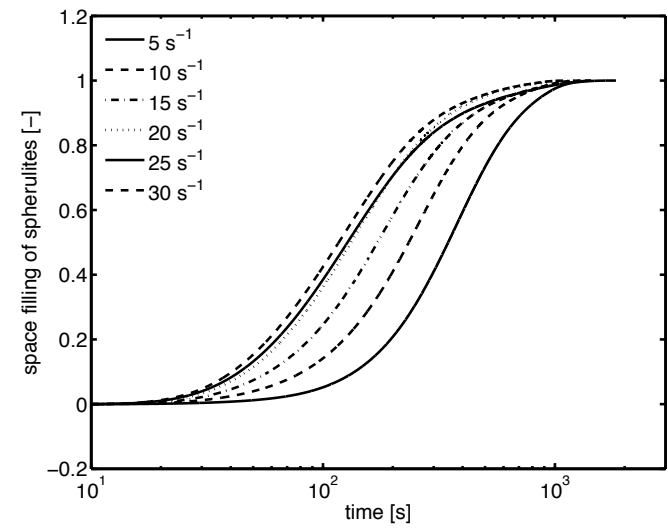

(a)

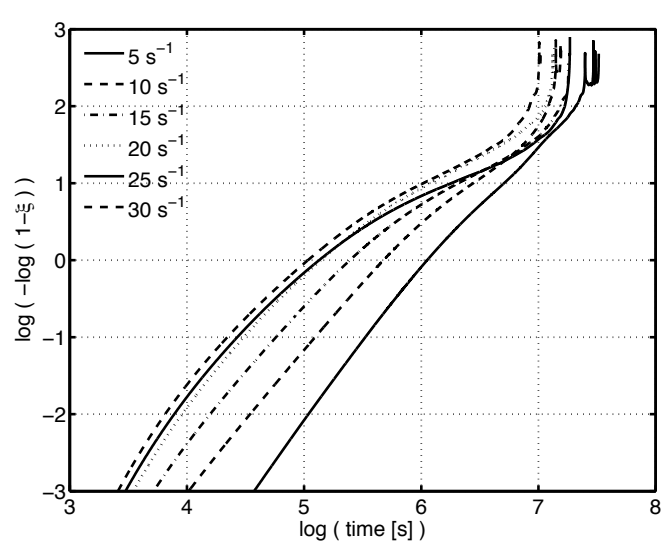

(b)

Figure 4: (a) Evolution of space filling for all shear rates. (b) Avrami plot of the crystallization of spherulites for all flow conditions.

\subsection{Rheology}

Figure 5 shows the evolution of the dynamic modulus for all flow rates. Due to the increase of nucleation density with shear rate, the dynamic modulus increases faster with increasing shear rate. However, the measured modulus increases at a much faster rate than the bulk crystallization kinetics. For the strongest flow condition, space filling reaches $50 \%$ at about 100 s (Fig. 4(a)). By that time the dynamic modulus is already close to its final value. Additionally, the first measurement point after shear already shows an increase of up to two orders of magnitude with respect to the modulus of the quiescent melt.

Apparently, the measured rheological properties do not correlate well to the bulk crystallization kinetics. This is easily explained from the way in which the modulus is determined in plate-plate rheometry. The device measures the torque, given by[27]

$$
T=2 \pi \int_{0}^{R} r^{2} \tau_{12}(r) \mathrm{d} r,
$$

with $T$ the torque, and $\tau_{12}$ the shear stress. From Eq. 7 it becomes obvious that the measured rheological properties are strongly dominated by the material at the edge of the sample (large $r$ ). We can quantify the factor with which torque increases if a layer near the outer edge of thickness 


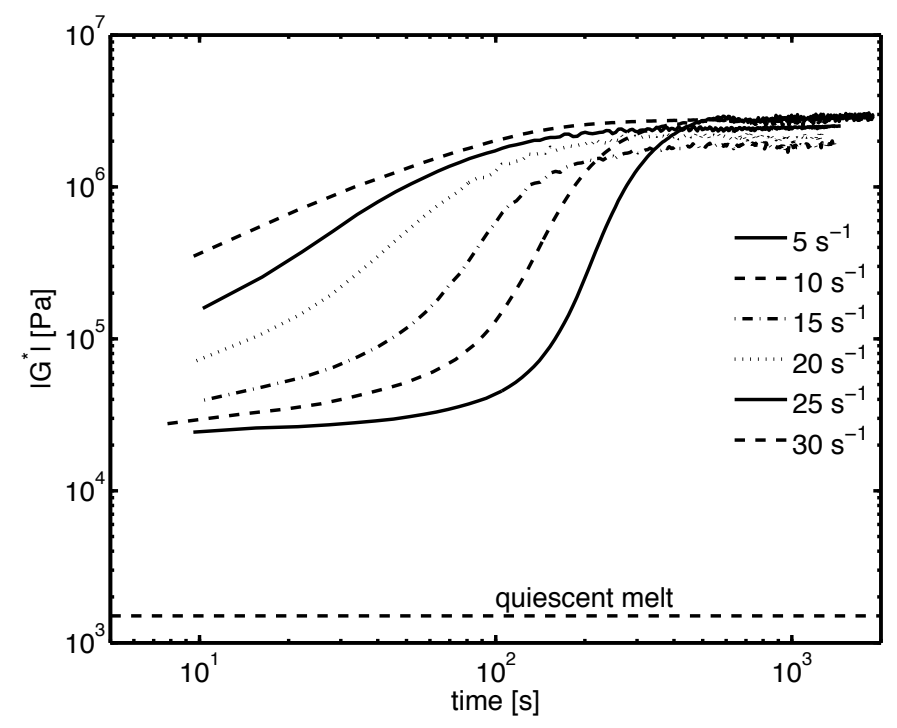

Figure 5: Evolution of the absolute value of the dynamic modulus after shear.

$d$ is solidified while the rest of the material is still in the molten state:

$$
\frac{T(d)}{T_{0}}=\frac{2 \pi \int_{0}^{R-d} r^{2} k\left(\frac{\dot{\gamma}_{w} r}{R}\right)^{n} \mathrm{~d} r+\mu \cdot 2 \pi \int_{R-d}^{R} r^{2} k\left(\frac{\dot{\gamma}_{w} r}{R}\right)^{n} \mathrm{~d} r}{2 \pi \int_{0}^{R} r^{2} k\left(\frac{\dot{\gamma}_{w} r}{R}\right)^{n} \mathrm{~d} r} .
$$

With $k=1.5 \cdot 10^{4}$ and $n=0.34$ power law parameters to determine the viscosity (see Appendix A), $\dot{\gamma}_{w}$ the shear rate at $r=R$, and $\mu=3000$ [28] the ratio between the modulus of the crystallized material and the modulus of the melt. $T_{0}$ and $T$ are respectively the measured torque for a sample fully consisting of a quiescent melt, and with a solidified layer with thickness $d$ near the edge of the sample. Because the measured modulus directly correlates to the torque, this is also the factor with which the measured modulus will increase. Figure 6(a) shows $\frac{T}{T_{0}}$ versus thickness of the crystallized layer. Because the term in the integral depends so strongly on $r$, even a very small layer will strongly increase the measured modulus. Figure 6(b) the first measurement point after flow with calculations from Eq. 8 with the layer thickness computed from the DSC signal (Fig. 3(b)). The measurements and calculations show excellent agreement, proving that indeed the layer experiencing edge effects has a tremendous influence in the rheological signal.

This result is an important indication that these kinds of measurements should be interpreted with great care; the rheological signal is representative of a small section of the material rather than the bulk, and experimental issues affecting this layer may severely bias experimental results. Vega et al. also observed this particular fact in a conventional rheometer [29], at shear rates of $60 \mathrm{~s}^{-1}$, which in our view is a strong indication that edge effects have occurred and that the rheological signal should be interpreted with great care. Such artefacts may however be more prevalent in the current experiments than in others, because it is not possible to properly clean the sample in the RheoDSC device. 


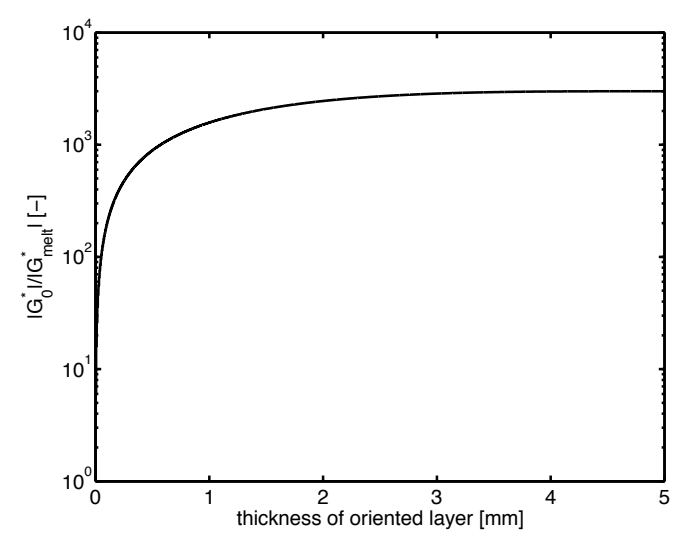

(a)

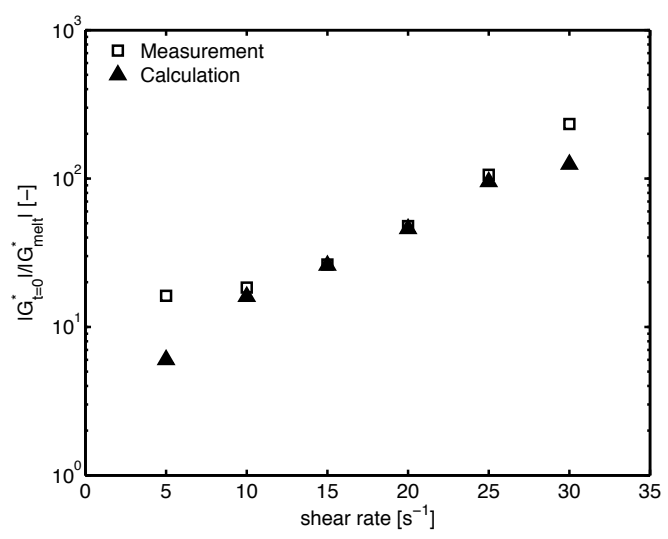

(b)

Figure 6: (a) Increase in measured rheology versus thickness of crystallized layer. (b) Increase with respect to melt rheology. Open symbols show the measurements, closed symbols show calculations with Eq. 8 where layer thickness is given by Eq. 5 .

\section{Conclusions}

Flow-induced structure formation in poly(1-butene) was investigated by means of short-term shear flow experiments in the RheoDSC device. It was found that the DSC signal reliably shows crystallization kinetics of the sample. For the investigated flow conditions, the large majority of the sample ( $>97 \%$ by volume) crystallized in the spherulitic morphology. The speed at which crystallization progressed, indicative of the number density of spherulites, consistently increased with shear rate. A small fraction of the sample ( $<3 \%$ by volume) crystallized in the shish-kebab morphology. Because the torque that is used to determine the modulus in plate-plate rheometry is strongly dominated by the material near the edges, this material strongly affects the measured rheology. The unique combination of rheometry and DSC in one device allowed us to quantify these effects. The results obtained indicate that rheological studies on flow-induced crystallization should be interpreted with care, especially at high shear rates when edge effects become important. The occurrence of these edge effects is signaled by an increase in modulus before the bulk of the sample crystallizes.

\section{Acknowledgements}

The European Soft Matter Infrastructure project (ESMI) is gratefully acknowledged for funding our measurement time at the Katholieke Universiteit Leuven (project no. E131100446). This project is supported by the Dutch Technology Foundation (STW), grant no. 08083 . 


\section{References}

[1] C. Carrot, J. Guillet, and K. Boutahar. Rheological behavior of a semi-crystalline polymer during isothermal crystallization. Rheologica Acata, 32:566--574, 1993.

[2] S. Vleeshouwers and H.E.H. Meijer. A rheological study of shear induced crystallization. Rheologica Acta, 35:391--399, 1996.

[3] J.W. Housmans, R.J.A. Steenbakkers, P.C. Roozemond, G.W.M. Peters, and H.E.H. Meijer. Saturation of pointlike nuclei and the transition to oriented structures in flow-induced crystallization of isotactic polypropylene. Macromolecules, 42:5728--5740, 2009.

[4] Z. Ma, R.J.A. Steenbakkers, J. Giboz, and G.W.M. Peters. Using rheometry to determine nucleation density in a colored system containing a nucleating agent. Rheologica Acta, 50: 909--915, 2011.

[5] G. Lamberti, G. W. M. Peters, and G. Titomanlio. Crystallinity and linear rheological properties of polymers. International Polymer Processing, 22(3):303--310, 2007.

[6] M. Derakhshandeh and S.V. Hatzikiriakos. Flow-induced crystallization of high-density polyethylene: the effects of shear and uniaxial extension. Rheologica Acta, 51:315--327, 2012.

[7] C. Hadinata, C. Gabriel, M. Ruellman, and H.M. Laun. Comparison of shear-induced crystallization behavior of PB-1 samples with different molecular weight distribution. Journal of Rheology, 49(1):327--349, 2005.

[8] C. Hadinata, D. Boos, C. Gabriel, E. Wassner, M. Ruellmann, N. Kao, and M. Laun. Elongation-induced crystallization of a high molecular weight isotactic polybutene-1 melt compared to shear-induced crystallization. Journal of Rheology, 51(2):195--215, 2007.

[9] P.C. Roozemond and G.W.M. Peters. Flow-enhanced nucleation of poly(1-butene): Model application to short-term and continuous shear and extensional flow. Journal of Rheology, 57:1633--1653, 2013.

[10] S. Acierno, S. Coppola, and N. Grizzuti. Effects of molecular weight distribution on the flow-enhanced crystallization of poly(1-butene). Journal of Rheology, 52(2):551--566, 2008.

[11] H. Fang, Y. Zhang, J. Bai, and Z. Wang. Shear-induced nucleation and morphological evolution for bimodal long chain branched polylactide. Macromolecules, 46:6555--6565, 2013.

[12] S. Liedauer, G. Eder, H. Janeschitz-Kriegl, P. Jerschow, W. Geymayer, and E. Ingolic. On the kinetics of shear-induced crystallization in polypropylene. International Polymer Processing, 8(3):236--244, 1993.

[13] R.J.A. Steenbakkers and G.W.M. Peters. Suspension-based rheological modeling of crystallizing polymer melts. Rheologica Acta, 47(5-6):643--665, 2008.

[14] P.C. Roozemond, V. Janssens, P. van Puyvelde, and G.W.M. Peters. Suspension-like hardening behavior of HDPE and time-hardening superposition. Rheologica Acta, 51: 97--109, 2012.

[15] S. Kiewiet, V. Janssens, H.E. Miltner, G.van Assche, P. van Puyvelde, and B. van Mele. RheoDSC: A hyphenated technique for the simultaneous measurement of calorimetric and rheological evolutions. Review of Scientific Instruments, 79(2, Part 1), 2008.

[16] V. Janssens, C. Block, G. van Assche, B. van Mele, and P. van Puyvelde. RheoDSC: design and validation of a new hybrid measurement technique. Journal of Thermal Analysis and Calometry, 98(3):675--681, 2009. 
[17] V. Janssens, C. Block, G. van Assche, B. van Mele, and P. van Puyvelde. RheoDSC analysis of the hardening of semi-crystalline polymers during quiescent isothermal crystallization. International Polymer Processing, 25(4):304--310, 2010.

[18] V. Janssens. RheoDSC: Development and validation of a combined rheometric and calorimetric measurement technique. PhD thesis, Katholieke Universiteit Leuven, 2010.

[19] J. Baert, P. van Puyvelde, and F. Langouche. Flow-induced crystallization of PB-1: From the low shear rate region up to processing rates. Macromolecules, 39:9215--9222, 2006.

[20] M. Avrami. Kinetics of phase change. I: General theory. The Journal of chemical physics, 7 (12):1103--1112, 1939.

[21] Fiorenza Azzurri. Melt crystallization and polymorphic transformation in isotactic poly(1-butene) based materials. PhD thesis, University of Genoa, 2003.

[22] J. Baert. Flow-induced crystallization of poly-1-butene. PhD thesis, Katholieke Universiteit Leuven, 2009.

[23] M. Seki, D.W. Thurman, J.P. Oberhauser, and J.A. Kornfield. Shear-mediated crystallization of isotactic polypropylene: The role of long chain-long chain overlap. Macromolecules, 35:2583--2594, 2002.

[24] P.C. Roozemond, Z. Ma, K. Cui, L. Li, and G.W.M. Peters. Multi-morphological crystallization in isotactic polypropylene: a quantitative model for parent-daughter crystallization kinetics. In preparation.

[25] O.O. Mykhaylyk, P. Chambom, R.S. Graham, J.P.A. Fairclough, P.D. Olmsted, and A.J. Ryan. The specific work of flow as a criterion for orientation in polymer crystallization. Macromolecules, 41:1901--1904, 2008.

[26] O.O. Mykhaylyk, P. Chambon, C. Impradice, J.P.A. Fairclough, N.J. Terrill, and A.J. Ryan. Control of structural morphology in shear-induced crystallization of polymers. Macromoleculus, 43:2389--2405, 2010.

[27] C.W. Macosko. Rheology: Principles, Measurements, and Applications. Wiley-VCH, 1994.

[28] L. Bove and M. R. Nobile. Shear-Induced Crystallization of Isotactic Poly(1-butene). Macromolecular Symposia, 185:135--147, 2002.

[29] J.F. Vega, D.G. Hristova, and G.W.M. Peters. Flow-induced crystallization regimes and rheology of isotactic polypropylene. Journal of Thermal Analysis and Calorimetry, 98: 655--666, 2009. 


\section{Appendix A : Viscous dissipation}

The total contribution to the heat flow from shear heating, obtained by integrating Eq. 2 over time, is shown in Fig. 7(a). The total heat per mass added to the system by shear heating can be calculated with

$$
\begin{aligned}
H & =\frac{1}{m} \int_{0}^{2 \pi} \int_{0}^{R} \int_{0}^{t_{s}} \sigma_{12} \dot{\gamma} r \mathrm{~d} \theta \mathrm{d} r \mathrm{~d} t \\
& =\frac{2 \pi t_{s}}{m} \int_{0}^{R} k\left(\frac{\dot{\gamma}_{w} r}{R}\right)^{n+1} r \mathrm{~d} r \\
& =\frac{2 \pi t_{s}}{m} \frac{k \dot{\gamma}_{w}^{n+1}}{R^{n+1}} \int_{0}^{R} r^{n+2} \mathrm{~d} r .
\end{aligned}
$$

Here $k$ and $n$ are parameters in a power-law representation of the viscosity of the material, as shown in Fig. 7 (b). From the above, it follows that the total heat added to the system should scale with $\dot{\gamma}^{n+1}$, shown by the dashed line in Fig. 7(a). This indicates that indeed the baseline that we determine for each experiment is a reasonable depiction of the dissipation of energy generated by shear heating.

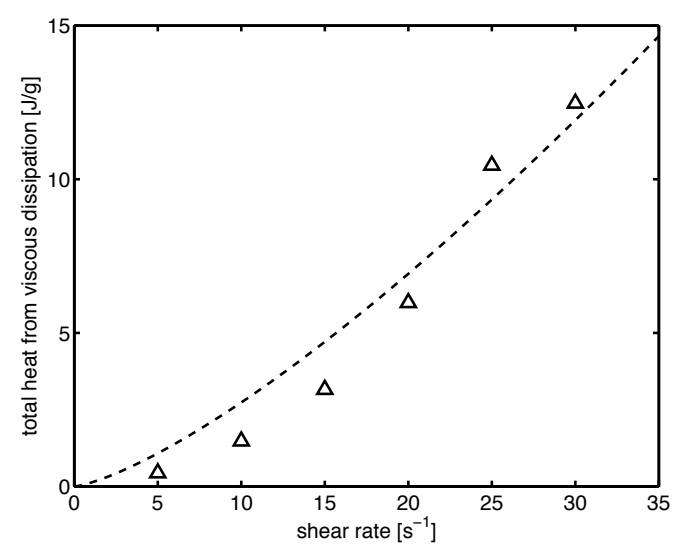

(a)

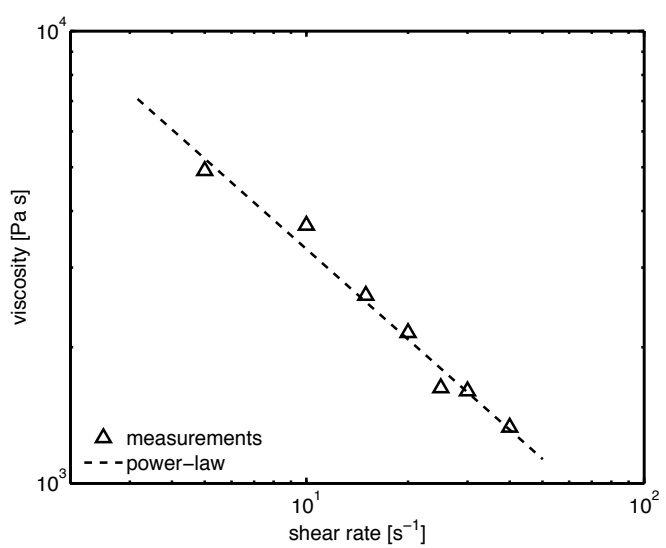

(b)

Figure 7: (a) Total contribution to heat flow from viscous dissipation. Line shows a relation of type $\Delta H \sim \dot{\gamma}^{n+1}$. (b) Steady-state viscosity versus shear rate. Line shows a power law $\eta=k \dot{\gamma}^{n-1}$ with $k=1.5 \cdot 10^{4}$ and $n=0.34$. 


\section{Appendix B : Average crystallization kinetics}

Because the shear rate is not homogeneous throughout the sample, neither is the flow-induced nucleation density. Therefore the crystallization kinetics measured with DSC are actually an average over the whole sample. Consequently, the Avrami equation for spherulites (Eq. 1) does not give a good representation of crystallization kinetics measured with DSC. In this Appendix we qualitatively show how this can result in exponents smaller than 3 in an Avrami plot.

The average crystallization kinetics of a cylindrical sample in which the nucleation density depends on shear rate, with crystallization kinetics in each point given by the Avrami equation for spherulites (Eq. 1) are given by

$$
\xi_{\text {avg }}(t)=\frac{2 \pi}{\pi R^{2}} \int_{0}^{R}\left[1-\exp \left(-\frac{4 \pi}{3} N(r) G^{3} t^{3}\right)\right] r \mathrm{~d} r,
$$

where $N(r)$ is the nucleation density distribution in radial direction. For different distribution functions, the average space filling and its corresponding Avrami plot are shown in Fig. 8. Indeed, the slope in the Avrami plot is smaller than 3. This is quite easy to understand: material near the edge of the sample, with high nucleation density, already reaches impingement whereas material in the center of the sample is just starting to crystallize. Therefore the average crystallization kinetics can not be described with a simple equation.

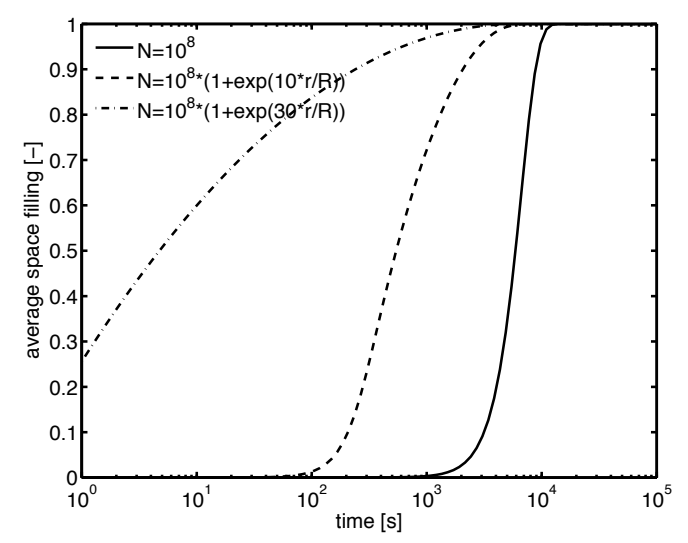

(a)

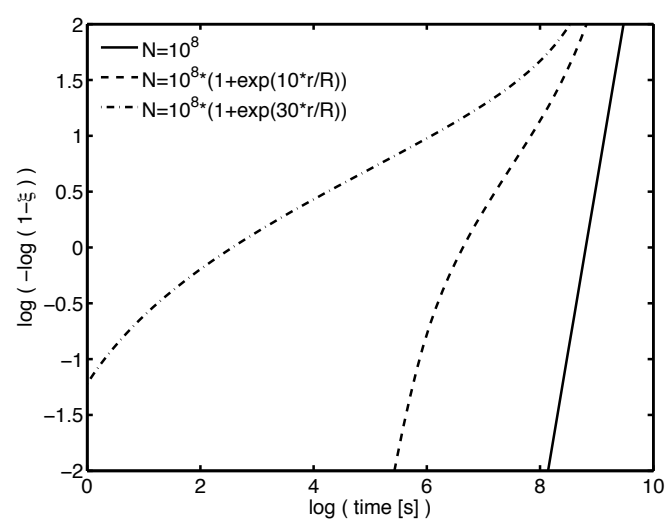

(b)

Figure 8: (a) Average crystallization kinetics for a sample with a distribution of nucleation density across radial direction. (b) Corresponding Avrami plot. 